University of South Carolina

Scholar Commons

$5-2008$

\title{
Spectrally Shaped Generalized MC-DS-CDMA with Dual Band Combining for Increased Diversity
}

Wenhui Xiong

David W. Matolak

University of South Carolina - Columbia, matolak@cec.sc.edu

Follow this and additional works at: https://scholarcommons.sc.edu/elct_facpub

Part of the Signal Processing Commons, and the Systems and Communications Commons

\section{Publication Info}

Postprint version. Published in IEEE Transactions on Wireless Communications, Volume 7, Issue 5, 2008, pages 1676-1686.

(C) IEEE Transactions on Wireless Communications, 2008, IEEE

Xiong, W., Matolak, D. (2008). Spectrally Shaped Generalized MC-DS-CDMA with Dual Band Combining for Increased Diversity. IEEE Transactions on Wireless Communications, 7(5), 1676-1686.

http://dx.doi.org/10.1109/TWC.2008.060744.

This Article is brought to you by the Electrical Engineering, Department of at Scholar Commons. It has been accepted for inclusion in Faculty Publications by an authorized administrator of Scholar Commons. For more information, please contact digres@mailbox.sc.edu. 


\title{
Spectrally Shaped Generalized MC-DS-CDMA with Dual Band Combining for Increased Diversity
}

\author{
Wenhui Xiong and David W. Matolak, Senior Member, IEEE
}

\begin{abstract}
A new multicarrier spread spectrum modulation scheme is proposed in this paper. This scheme uses sinusoidal chip waveforms to shape the spectrum of each subcarrier of a multicarrier direct sequence spread spectrum (DS-SS) signal. As a result, each subcarrier has two distinct spectral lobes, one a lower sideband (LSB) and the other an upper sideband (USB). By properly selecting the parameters of the sinusoidal chip waveforms, the two sideband signals can be made to undergo independent fading in a dispersive fading channel. These two independently-faded sideband signals, when combined at the receiver, provide diversity gain to the system. Our analysis and simulation results show that by properly selecting the chip waveform parameter and the intersubcarrier frequency separation, the bit error ratio (BER) performance of the proposed scheme is superior to that of the conventional MC-DS-CDMA system in dispersive fading channels. In addition, spectral sidelobes are naturally reduced by our scheme.
\end{abstract}

Index Terms-Direct sequence spread spectrum, multicarrier.

\section{INTRODUCTION}

D IRECT sequence spread spectrum (DS-SS) is widely used in both civilian and military applications for its well known advantages, such as multiple access, low probability of interception and resistance to interference [1]. One of the attractive features of DS-SS, when used in a wireless environment, is that the wide signal bandwidth helps the receiver to resolve channel echoes, or multipath. These echoes, when combined in a maximal ratio combining (MRC) fashion, provide the system so called multipath diversity, which improves system error probability performance.

Recent research has addressed the combination of spread spectrum and multicarrier modulation, resulting in the multicarrier spread spectrum (MC-SS) signaling schemes [2]. There are (at least) three types of multicarrier spread spectrum schemes: MC-CDMA, MC-DS-CDMA, and MT-DS-CDMA. In MC-CDMA [3-5], the spreading is performed "in the frequency domain," where the chips of the DS spreading signal are transmitted via different subcarriers, yielding frequency diversity. For MC/MT-DS-CDMA systems [6-10], spreading is performed "in the time domain," which brings multipath diversity to the system when channel echoes are combined via MRC. The only difference between the MC-DS-CDMA and

Manuscript received September 24, 2006; revised September 15, 2007 and November 22, 2007; accepted January 10, 2008. The associate editor coordinating the review of this paper and approving it for publication was A. Stefanov.

W. Xiong is with Qualcomm Inc., 5775 Morehouse Dr. San Diego CA 92121 (e-mail: wenhuix@qualcomm.com).

D. W. Matolak is with Ohio University, School of Electrical Engineering and Computer Science, Athens, OH 45701 (e-mail: matolak@ohiou.edu).

Digital Object Identifier 10.1109/TWC.2008.060744.
MT-DS-CDMA schemes is the frequency separation between adjacent subcarriers [2]. For MT-DS-CDMA the frequency separation is the data rate of each subcarrier, whereas the frequency separation for MC-DS-CDMA is the chip rate of each subcarrier. It was also shown in [11] that these two types of schemes are actually special cases of a generalized multicarrier DS-SS signaling scheme, whose performance in dispersive fading channels can be analyzed under a common frame work. In [12] the authors proposed an MC-SS system that spreads the signal in both time and frequency domains, where the same data bit is DS-SS spread, and transmitted through orthogonal subcarriers. By spreading in both time and frequency domains, the system can obtain higher diversity order at the price of lower system throughput compared with the MC-DS-CDMA type systems we are considering.

The spectral sidelobes of any transmitted signal may act as interference to signals in adjacent bands, and traditionally these sidelobes are suppressed via filtering, e.g., using a raised cosine filter. In [13], the MC-SS sidelobe levels were reduced by concentrating more energy in the central, or main lobe. This concentration can be achieved by adjusting the data rate, chip rate and frequency separations between adjacent subcarriers. In addition to sidelobe suppression, the transmission scheme in [13] can shape the spectrum of the transmitted signal by appropriate selection of the aforementioned parameters. Spectral shaping can also be achieved using different chip waveforms. In [14] the spectrum of a single carrier DS-CDMA signal was shaped to have a flat spectrum within a specified band by using a band limited chip waveform. The purpose of this band limited chip waveform was to minimize multiple access interference (MAI) in a CDMA application when the channel is the additive white Gaussian noise (AWGN) channel. Following this work, authors in [15] and [16] used numerical methods to find the optimal time-limited chip waveform that minimizes both the MAI and the out-of-band signal energy, again in the AWGN channel. In [17], the authors investigated the bit error performance of generalized MC-DS-CDMA [11] with different chip waveforms, i.e., rectangular, half-sine, and raised cosine chip waveforms. Their results showed that for a given intersubcarrier spacing there exists an optimal choice of chip waveform. The effects of chip waveform selection on the performance of MC-DS-CDMA were investigated in [18]. The author demonstrated that the performance of MC-DS-CDMA systems is insensitive to the chip waveform shaping, but this investigation, as well as the work done by [17], did not include our two-sideband approach.

In this paper, we propose a new multicarrier modulation scheme that uses sinusoidal chip waveforms to shape the 


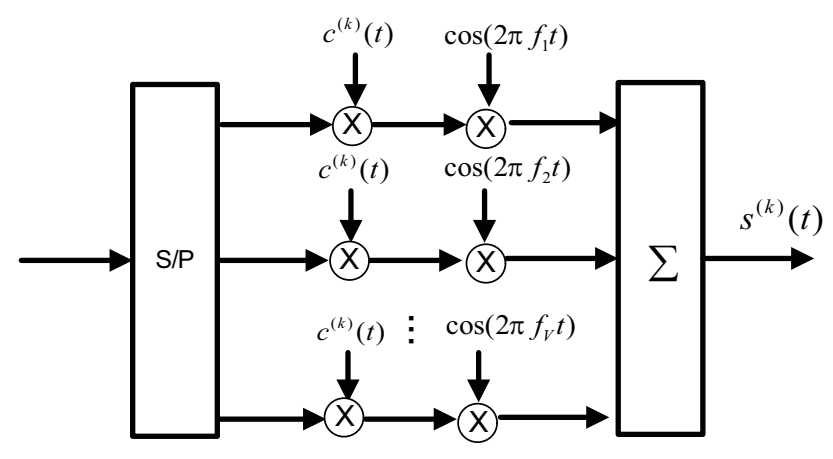

Fig. 1. Transmitter block diagram for user $\mathrm{k}$.

spectrum of each subcarrier. For a given subcarrier, the chip waveform of our proposed scheme shapes the transmitted signal spectrum to have two distinct lobes, a lower sideband (LSB) and an upper sideband (USB). With properly selected chip waveform parameters, the separation between these two lobes can be wider than the channel's coherence bandwidth, resulting in the two sidebands undergoing uncorrelated fading. This uncorrelated fading of the LSB and USB can be utilized to greatly improve performance by virtue of diversity.

This paper is organized as follows: Section II introduces the proposed system transceiver waveforms and the Nakagami$\mathrm{m}$ dispersive fading channel model we employ. Section III provides the analysis of receiver decision statistics required for error probability estimation. In Section IV we provide numerical results and discussion, and Section V contains a summary and conclusions.

\section{SySTEM MODEL}

\section{A. Transmitted Signal}

A block diagram of the transmitter is shown in Fig.1. For simplicity of exposition, we use binary phase shift-keying (BPSK) modulation, but results can easily be extended to higher order PSK. As shown in the figure, user $k$ 's binary data stream is first serial to parallel converted to form $V$ parallel data streams each with bit duration of $T_{b}$. Each of the $V$ branches of the data stream is spread by the (same) spreading waveform with chip duration of $T_{c}$. As in any CDMA system, the spreading code is used to distinguish users, and the same random long code is used for each subcarrier of a given user signal. There are $K$ user signals present. After spreading, the chips of each subcarrier modulate the subcarrier sinusoids, and the transmitted signal is formed via summing the signals of all subcarriers. The transmitted signal of user $k$ is

$$
\begin{aligned}
s^{(k)}(t)= & \sqrt{\frac{2 E_{b}}{T_{b}}} \sum_{i=1}^{V} \sum_{n=-\infty}^{\infty} d_{i}^{(k)}(\lfloor n / N\rfloor) \\
& \times c^{(k)}(n) p\left(t-n T_{c}\right) \cos \left(2 \pi f_{i} t\right)
\end{aligned}
$$

where $E_{b}, T_{b}$ are respectively the bit energy and bit duration of each subcarrier, $d_{i}^{(k)}(n) \in\{ \pm 1\}$ is user $k$ 's $n$th transmitted data bit on the $i$ th subcarrier, $\lfloor x\rfloor$ is the integer part of $x$, and $N=T_{b} / T_{c}$ is the processing gain on any subcarrier (equal for all $k$ and $i$ ). We also define $c^{(k)} \in\{ \pm 1\}$ as the $m$ th chip of user $k$ 's spreading code, $p(t)=\sqrt{2} \sin \left(J \pi t / T_{c}\right)$ over the interval $0 \leq t \leq T_{c}$ is the chip waveform with integer $J \in$ $\{2,3, \cdots\}$ a selectable parameter, and $f_{i}$ is the ith subcarrier frequency. ${ }^{1}$

Most of our results employ odd values of $J$. In addition, we use the same spreading waveform for all subcarriers, i.e., $c^{(k)}(t)=\sum c^{(k)}(n) p\left(t-n T_{c}\right)$ is the same for all subcarriers of user $k$. The frequency separation between adjacent subcarriers is selected such that any two subcarriers are orthogonal. Specifically, when $i \neq j$ this requires

$$
\int_{0}^{T_{b}} \sum_{n=0}^{N-1} p^{2}\left(t-n T_{c}\right) \cos \left(2 \pi f_{i} t\right) \cos \left(2 \pi f_{j} t\right) d t=0
$$

As shown in Appendix I, (2) is satisfied when $\Delta f_{i, j}=$ $\left|f_{i}-f_{j}\right|=\lambda / T_{b}$ and $\Delta f_{i, j} \neq J$ where $\lambda$ is an integer. In this work, we allow $\lambda$ to vary from 1 to $N$; this corresponds, by analogy, to configuring the system to vary from MT-DSCDMA to MC-DS-CDMA (as in [11]). It is worth noting that when $\lambda=1$ some subcarriers may no longer be orthogonal to each other. However, since the dispersive channel destroys the orthogonality between subcarriers in any case, we still investigate the error performance of the $\lambda=1$ case. The transmitted signal can also be expressed by a summation of the LSB and USB of each subcarrier signal as

$$
s^{(k)}(t)=\sum_{i=1}^{V}\left[s_{i, L}^{(k)}(t)+s_{i, U}^{(k)}(t)\right]
$$

where each sideband signal of subcarrier $i$ of user $k$ can be expressed by

$$
\begin{aligned}
s_{i, X}^{(k)}(t)=\sqrt{\frac{E_{b}}{2 T_{b}}} & \sum_{n=-\infty}^{\infty} d_{i}^{(k)}(\lfloor n / N\rfloor) c^{(k)}(n) \\
& \times\left[p\left(t-n T_{c}\right) \cos \left(2 \pi f_{i} t\right)\right. \\
& \left. \pm \hat{p}\left(t-n T_{c}\right) \sin \left(2 \pi f_{i} t\right)\right]
\end{aligned}
$$

where $\hat{p}\left(t-n T_{c}\right)$ is the Hilbert transform of the sinusoidal chip waveform $p(t)=\sqrt{2} \sin \left(J \pi t / T_{c}\right)$, and $X=L$ or $U$ represents the LSB or USB of the transmitted signal. With some algebraic simplification, we can easily show that $\hat{p}(t)=$ $-\sqrt{2} \cos \left(J \pi t / T_{c}\right)$ for $0 \leq t \leq T_{c}$. Upon substituting the chip waveform $p(t)$ and $\hat{p}(t)$ into (4), we can write the sideband signal of subcarrier $i$ as

$$
\begin{array}{r}
s_{i, X}^{(k)}(t)=\sqrt{\frac{E_{b}}{T_{b}}} \sum_{n=-\infty}^{\infty} d_{i}^{(k)}(\lfloor n / N\rfloor) c^{(k)}(n) \\
\times E_{i, X}\left(t-n T_{c}, 0\right)
\end{array}
$$

In (5), $E_{i, X}(t, \varphi)$ is the passband chip waveform for subcarrier $i$, which is

$$
E_{i, X}(t, \varphi)=\left\{\begin{array}{cc}
\sin \left[\left(\frac{J}{T_{c}} \mp f_{i}\right) \pi t+\varphi\right] & 0 \leq t \leq T_{c} \\
0 & \text { otherwise }
\end{array}\right.
$$

In (6), $\varphi$ is the phase of the passband chip waveform for subcarrier $i$, the minus sign represents the passband chip

\footnotetext{
${ }^{1}$ Note that the use of rectangular chip waveforms is different from a simple upconversion of the baseband rectangular chip waveform subcarrier signal to some carrier frequency. Specifically, when $J$ is odd, phase discontinuities are enforced at chip boundaries where they otherwise would not be for an upconversion (when two consecutive chip pulses $c^{(k)}(m), c^{(k)}(m+1)$ are equal).
} 


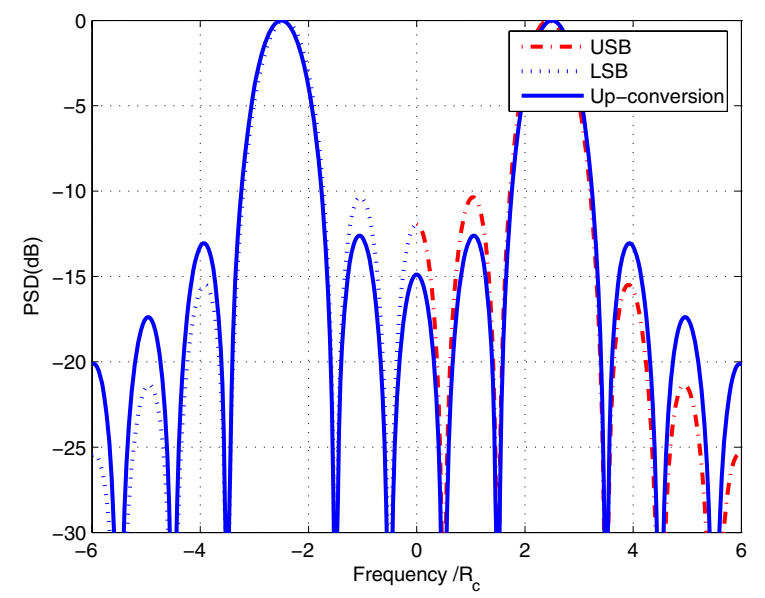

Fig. 2. Example PSD of one subcarrier for proposed scheme with $J=5$.

waveform for the LSB signal, and the plus sign represents the chip waveform for the USB signal. ${ }^{2}$ It is worth noting that by expressing each subcarrier signal as a sum of LSB and USB component signals, the nature of each subcarrier signal is not changed, and thus orthogonality between any two subcarriers is maintained.

\section{B. Channel Model}

Each subcarrier of our proposed scheme is a DS-SS signal with its power spectral density (PSD) determined by the Fourier transform of its chip waveform [19]. For the sinusoidal chip waveform $p(t)$, the baseband PSD of one subcarrier is given by

$$
\begin{aligned}
\psi(f)= & \frac{E_{b}}{N}\left(\frac{2 J}{4 \pi\left(T_{c} f\right)^{2}-\pi J^{2}}\right)^{2} \\
& \times\left[1-(-1)^{J} \cos \left(2 \pi T_{c} f\right)\right]
\end{aligned}
$$

An example PSD of one subcarrier using the sinusoidal chip waveform with $J=5$ is shown in Fig. $2^{3}$. The separation between the peaks of the two sidelobes is approximately $J / T_{c}$, and the null-null bandwidth of each sideband is $2 / T_{c}$. Therefore, two sideband signals incur uncorrelated fading when the chip waveform parameter is properly chosen, i.e., the frequency separation between the two mainlobes of the LSB and USB is greater than the channel coherence bandwidth. We assume that the channel for either LSB or USB, for the $i$ th subcarrier of user $k$, is a dispersive Nakagami- $m$ channel with impulse response given by

$$
h_{i, X}^{(k)}(t)=\sum_{l=0}^{M-1} \alpha_{i, l}^{(k, X)} \exp \left(-j \theta_{i, l}^{(k, X)}\right) \delta\left(t-\tau_{i, l}^{(k, X)}\right)
$$

\footnotetext{
${ }^{2}$ The representation of (3) and (5) can be directly obtained by multiplying $\sqrt{2} \sin \left(J \pi t / T_{c}\right)$ and $\cos \left(2 \pi f_{i} t\right)$.

${ }^{3}$ As can be observed in this figure, the PSD of our proposed scheme for one subcarrier is different from that of the subcarrier signal that is up-converted by the carrier waveform $\sin \left(5 \pi t / T_{c}\right)$. Specifically, despite the same mainlobe shapes, the sidelobe levels of the two schemes-using our chip waveform and conventional up-conversion-are different. As previously noted, this is because when $J$ is odd, phase discontinuities are enforced at chip boundaries where they otherwise would not be for an upconversion (when two consecutive chip pulses $c^{(k)}(m), c^{(k)}(m+1)$ are equal).
}

where $X=L$ or $U$ again represents LSB or USB. In (8) $M$ is the number of resolvable channel paths given by [6]

$$
M=\left\lfloor B W / B_{c}\right\rfloor+1
$$

where $B W=2 / T_{c}$ is the baseband null-to-null bandwidth of either sideband. In (8), $\alpha_{i, l}^{(k, X)}, \theta_{i, l}^{(k, X)}$, and $\tau_{i, l}^{(k, X)}$ are respectively the amplitude, phase shift, and delay of the $l$ th tap for user $k$ 's LSB or USB signal on subcarrier $i$. Under the assumption of uncorrelated scattering (US) and independent fading for the LSB and USB signals, the phase shifts of different user's different paths of different subcarriers are independent identically distributed (i.i.d.) random variables (RVs) uniformly distributed within $[0,2 \pi)$. Similarly, the delays for different $i, l, k$ and $X$ are i.i.d. random variables uniformly distributed within the interval $\left[0, T_{b}\right)$. The tap amplitudes for different sidebands, different taps and different users are assumed to be independent Nakagami- $m$ RVs. We use the Nakagami model because of its flexibility and ability to model a wide range of fading conditions [11]. The probability density function (pdf) of a Nakagami- $m$ RV is given by

$$
p_{R}(r)=\frac{2}{\Gamma(m)}\left(\frac{m}{\Omega}\right)^{m} r^{2 m-1} e^{-m r^{2} / \Omega}
$$

where $\Gamma()$ is the gamma function, $\Omega=E\left[r^{2}\right]$ is the average energy, and $m>0$ is the fading parameter, which characterizes the channel conditions. Specifically, when $m=1 / 2$ the Nakagami- $m$ distribution becomes the one sided Gaussian distribution; for $m=1$, it becomes the well known Rayleigh distribution, and when $m \rightarrow \infty$, it becomes an impulse, or a non-fading distribution.

In this paper, the power delay profile (PDP)of the channels between the transmitter and receiver for both sideband signals of all users' subcarriers are assumed to be identical, i.e., $\Omega_{i, l}^{(k, X)}=\Omega_{l}$ for $X=L, U, i=1,2, \cdots V$, and $k=$ $1,2, \cdots K$. In addition, the PDP shape is assumed to be exponentially decaying [11], i.e., the average power of the $l$ th tap is given by $\Omega_{l}=\Omega_{0} e^{-\eta l}$ where $\eta$ is the decay factor, and the total power of the channel is normalized to be unity, i.e., $\sum_{l=0}^{M-1} \Omega_{l}=1$.

\section{Receiver Model}

Assuming we have $K$ asynchronous users with identical system configurations (same number of subcarriers, processing gain and chip waveforms for each subcarrier), and independent channels for both the LSB and USB signals of all subcarriers of all users, the received signal is

$$
r(t)=\sum_{k=1}^{K} \sum_{i=1}^{V}\left[r_{i, L}^{(k)}(t)+r_{i, U}^{(k)}(t)\right]+n(t)
$$

where $n(t)$ is the AWGN with double sided PSD $N_{0} / 2 \mathrm{~W} / \mathrm{Hz}$. The received LSB or USB signal of the $i$ th subcarrier of user $k, r_{i, X}^{(k)}$, is given by

$$
\begin{array}{r}
r_{i, X}^{(k)}=\sqrt{\frac{E_{b}}{T_{b}}} \sum_{l=0}^{M-1} \sum_{n=-\infty}^{\infty} \alpha_{i, l}^{(k, X)} d_{i}^{(k)}(\lfloor n / N\rfloor) c^{(k)}(n) \\
\times E_{i, X}\left(t-n T_{c}, \phi_{i, l}^{(k, X)}\right)
\end{array}
$$




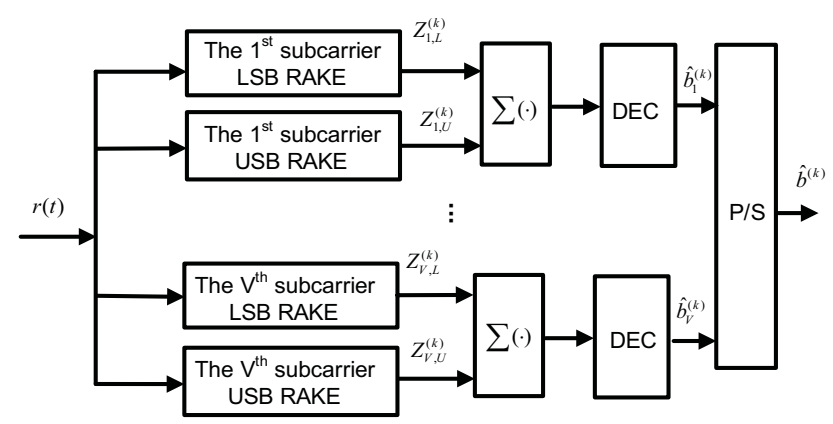

Fig. 3. Receiver block diagram for user k.

where $\phi_{i, l}^{(k, X)}=2 \pi f_{i} \tau_{i, l}^{(k, X)}-\theta_{i, l}^{(k, X)}$ is the composite phase, also uniformly distributed within $[0,2 \pi)$.

We assume that the receiver is capable of estimating the channel perfectly, i.e., the receiver can estimate the amplitude, delay, and phase of each multipath echo. Thus, similar to the conventional CDMA RAKE receiver, with each finger locked to one path, two RAKEs are needed for one subcarrier. It is also worth noting that when the local reference signal of each RAKE finger is $c^{(k)}(n) E_{i, X}\left(t-n T_{c}, \phi_{i, l}^{(k, X)}\right)$, no additional signal processing component is needed before these RAKE receivers. The receiver block diagram for user $\mathrm{k}$ is shown in Fig. 3, where two RAKEs, one for each sideband, are used for each subcarrier. After maximal ratio combining (MRC) the RAKE fingers, the outputs of the two RAKEs are combined to form the decision metric for the given subcarrier. Finally, a hard decision is made via the sign of the combined output, and these detected bits are parallel to serial converted to form the detected bit stream. The decision metric for user $k$ 's $j$ th subcarrier is

$$
Z_{j}^{(k)}=\sum_{X} \sum_{q=0}^{M-1} Z_{j, q}^{(k, X)}
$$

where $Z_{j, q}^{(k, X)}$ is the correlator output of the qth RAKE finger of the $j$ th subcarrier of user $k$.

\section{Performance AnAlysis}

\section{A. Corrrelator Output}

Without loss of generality, we assume the phase and delay of the $q$ th path of user $k$ 's subcarrier $j$ are zero, and the delays and phases of other channel taps are relative to those of path $q$. Thus, user $k$ 's $q$ th RAKE finger correlator output for subcarrier $j$ can be separated into four terms

$$
\begin{aligned}
Z_{j, q}^{(k, X)} & =\int_{0}^{T_{b}} r(t) \alpha_{j, q}^{(k, X)} \sum_{n=-\infty}^{\infty} c^{(k)}(n) E_{j, X}\left(t-n T_{c}\right) d t \\
& =D+n+I_{1}+I_{2}+I_{3}
\end{aligned}
$$

where $D=\sqrt{E_{b} T_{b}} d_{j}^{(k)}(0) / 2$ is the desired signal term, $n$ is the AWGN sample with zero mean and variance $N_{0} T_{b} / 4$. The terms $I_{1}$ and $I_{2}$ are the interferences from the same subcarrier (both same and different users) due to the channel dispersion, and $I_{3}$ is the interference from other subcarriers. The interference from the same subcarrier of the same user,
$I_{1}$, is given by

$$
\begin{aligned}
I_{1}= & \sqrt{\frac{E_{b}}{T_{b}}} \sum_{l=0, l \neq q}^{M=1} \alpha_{j, q}^{(k, X)} \alpha_{j, l}^{(k, X)} \beta_{j, j}^{(k, k)}\left(\tau_{j, l}^{(k, X)}, \phi_{j, l}^{(k, X)}\right) \\
& +\sqrt{\frac{E_{b}}{T_{b}}} \sum_{l=0}^{M=1} \alpha_{j, q}^{(k, X)} \alpha_{j, l}^{(k, Y)} \hat{\beta}_{j, j}^{(k, k)}\left(\tau_{j, l}^{(k, Y)}, \phi_{j, l}^{(k, Y)}\right)
\end{aligned}
$$

where we denote the correlation function between the same sideband waveform from user $v$ 's subcarrier $i$ and that of the user $k$ 's subcarrier $j$ by $\beta_{i, j}^{(v, k)}$, and similarly $\hat{\beta}_{i, j}^{(v, k)}$ is defined for the correlation function between different sideband signals. Note that since the LSB and USB signal undergo independent fading, the interference from the qth path of the other sideband signal (indicated as $Y$ ) is not excluded from the summation of the second term of (15).

Similarly, the interference from the same subcarriers of different users, $I_{2}$, and the interference from other subcarriers of all users, $I_{3}$, are respectively given by

$$
\begin{gathered}
I_{2}=\sqrt{\frac{E_{b}}{T_{b}}} \sum_{v=1, v \neq k}^{K} \sum_{l=0}^{M=1} \alpha_{j, q}^{(k, X)} \alpha_{j, l}^{(v, X)} \\
\times \beta_{j, j}^{(v, k)}\left(\tau_{j, l}^{(v, X)}, \phi_{j, l}^{(v, X)}\right) \\
+\sqrt{\frac{E_{b}}{T_{b}}} \sum_{v=1, v \neq k}^{K} \sum_{l=0}^{M=1} \alpha_{j, q}^{(k, X)} \alpha_{j, l}^{(v, Y)} \\
\times \hat{\beta}_{j, j}^{(v, k)}\left(\tau_{j, l}^{(v, Y)}, \phi_{j, l}^{(v, Y)}\right) \\
I_{3}=\sqrt{\frac{E_{b}}{T_{b}} \sum_{v=1}^{K}} \sum_{i=1, i \neq j}^{V} \sum_{l=0}^{M=1} \alpha_{j, q}^{(k, X)} \alpha_{i, l}^{(v, X)} \\
\times \sqrt{\frac{E_{b}}{T_{b}}} \sum_{v=1}^{K} \sum_{i=1, i \neq j}^{V} \sum_{l=0}^{V, k)}\left(\tau_{i, l}^{(v, X)}, \phi_{i, l}^{(v, X)} \alpha_{j, q}^{(k, X)} \alpha_{i, l}^{(v, Y)}\right. \\
\quad \times \hat{\beta}_{i, j}^{(v, k)}\left(\tau_{i, l}^{(v, Y)}, \phi_{i, l}^{(v, Y)}\right)
\end{gathered}
$$

where the $\beta_{i, j}^{(v, k)}$, and $\hat{\beta}_{i, j}^{(v, k)}$ functions in (15)-(17) are defined as (shown on the top of next page)

\section{B. Statistics of Decision Metric}

The statistics of the desired signal term and AWGN sample term were already given, so here we derive the statistics of the interference terms. The interference terms are assumed to be Gaussian random variables. This approximation is validated via our simulations shown in Section IV. In this section, we investigate the statistics of the cross correlation functions $\left(\beta_{i, j}(k, v)\right.$ and $\left.\hat{\beta}_{i, j}(k, v)\right)$ between the different subcarriers and different users. It is worth nothing that the cross correlation functions between the same subcarriers of different users can be obtained by equating the user indices $k$ and $v$; similarly, the cross correlation between the different multipath echoes of the given subcarrier from the same user can be found by letting $i=j$ and $v=k$. Following the method given in [13], we represent the delay $\tau$ in (18) and (19) as

$$
\tau=S T_{c}+\varepsilon
$$




$$
\begin{aligned}
& \beta_{i, j}^{(v, k)}(\tau, \phi)=\int_{0}^{T_{b}} \sum_{n=-\infty}^{\infty} d_{i}^{(v)}(\lfloor n / N\rfloor) c_{i}^{(v)}(n) E_{i, X}\left(t-n T_{c}-\tau, \phi\right) \sum_{m=0}^{N-1} c_{j}^{(k)}(m) E_{j, X}\left(t-n T_{c}, 0\right) \\
& \hat{\beta}_{i, j}^{(v, k)}(\tau, \phi)=\int_{0}^{T_{b}} \sum_{n=-\infty}^{\infty} d_{i}^{(v)}(\lfloor n / N\rfloor) c_{i}^{(v)}(n) E_{i, Y}\left(t-n T_{c}-\tau, \phi\right) \sum_{m=0}^{N-1} c_{j}^{(k)}(m) E_{j, X}\left(t-n T_{c}\right) d t
\end{aligned}
$$

where $S$ is an integer given by $S=\left\lfloor\tau / T_{c}\right\rfloor$ and $\varepsilon=\tau-S T_{c}$ is the fractional chip part of $\tau$, uniformly distributed in $\left[0, T_{c}\right)$. This representation of the $\tau$ allows $\beta_{i, j}(k, v)$ and $\hat{\beta}_{i, j}(k, v)$ to be expressed as

$$
\begin{aligned}
\beta_{i, j}^{(v, k)}(\tau, \phi)= & d_{i}^{(v)}(-1) R_{i, X ; j, X}^{(v, k)}(\tau, \phi) \\
& +d_{i}^{(v)}(0) \hat{R}_{i, X ; j, X}^{(v, k)}(\tau, \phi) \\
\hat{\beta}_{i, j}^{(v, k)}(\tau, \phi)= & d_{i}^{(v)}(-1) R_{i, Y ; j, X}^{(v, k)}(\tau, \phi) \\
& +d_{i}^{(v)}(0) \hat{R}_{i, Y ; j, X}^{(v, k)}(\tau, \phi)
\end{aligned}
$$

where $R_{i, Y ; j, X}^{(v, k)}$ and $\hat{R}_{i, Y ; j, X}^{(v, k)}$ are two partial correlation functions with definitions given by

$$
\begin{aligned}
R_{i, Y ; j, X}^{(v, k)}(\tau, \phi) & =\sum_{m=0}^{S} c^{(k)}(m) c^{(v)}(m-S-1) \\
& \times \int_{0}^{\varepsilon} E_{i, Y}(t-\varepsilon, \phi) E_{j, X}(t, 0) d t \\
& +\sum_{m=0}^{S-1} c^{(k)}(m) c^{(v)}(m-S) \\
\times & \int_{\varepsilon}^{T_{c}} E_{i, Y}(t-\varepsilon, \phi) E_{j, X}(t, 0) d t \\
\hat{R}_{i, Y ; j, X}^{(v, k)}(\tau, \phi)= & \sum_{m=S+1}^{N-1} c^{(k)}(m) c^{(v)}(m-S-1) \\
& \times \int_{0}^{\varepsilon} E_{i, Y}(t-\varepsilon, \phi) E_{j, X}(t, 0) d t \\
& \times \int_{\varepsilon}^{T_{c}} E_{i, Y}(t-\varepsilon, \phi) E_{j, X}(t, 0) d t
\end{aligned}
$$

Since we assume the data symbols and the spreading codes are random and binary, the two partial correlation functions of (23) and (24) are zero mean, and their variances are easy to evaluate, yielding,

$$
\begin{aligned}
& \operatorname{var}\left[\beta_{i, j}^{(v, k)}(\tau, \phi)\right]=2 N \operatorname{var}\left[\int_{0}^{\varepsilon} E_{i, X}(t-\varepsilon, \phi) E_{j, X}(t, 0) d t\right] \\
& \operatorname{var}\left[\hat{\beta}_{i, j}^{(v, k)}(\tau, \phi)\right]=2 N \operatorname{var}\left[\int_{0}^{\varepsilon} E_{i, Y}(t-\varepsilon, \phi) E_{j, X}(t, 0) d t\right]
\end{aligned}
$$

The derivation of the variances in (25) and (26) is given in Appendix II. With the variance of (25) and (26), the variances of the interference terms are

$$
\operatorname{var}\left[I_{1}\right]=\frac{E_{b} T_{b}}{N}\left[\frac{M-1}{12 M}+\frac{1}{8 J^{2} \pi^{2}}\right]\left(\alpha_{j, q}^{(k, X)}\right)^{2}
$$

$$
\begin{gathered}
\operatorname{var}\left[I_{2}\right]=(K-1) \frac{E_{b} T_{b}}{N}\left[\frac{1}{12}+\frac{1}{8 J^{2} \pi^{2}}\right]\left(\alpha_{j, q}^{(k, X)}\right)^{2} \\
\operatorname{var}\left[I_{3}\right]=\frac{K E_{b} T_{b}}{N} \sum_{\substack{j=1 \\
j \neq i}}^{V}\left(\operatorname{var}\left[I_{X}\left(\Delta f_{i, j} T_{c}\right)\right]\right. \\
\left.+\operatorname{var}\left[I_{Y}\left(\Delta f_{i, j} T_{c}\right)\right]\right)\left(\alpha_{j, q}^{(k, X)}\right)^{2}
\end{gathered}
$$

The definitions of $I_{X}$ and $I_{Y}$ and their variances are given in Appendix II. The mean and variance of the correlator output of the decision metric are thus

$$
\begin{array}{r}
\mathrm{E}\left[Z_{j}^{(v)}\right]=\frac{1}{2} \sqrt{E_{b} T_{b}} d(0) \sum_{X} \sum_{q=0}^{M-1}\left[\left(\alpha_{j, q}^{(k, X)}\right)^{2}\right] \\
\operatorname{var}\left[Z_{j}^{(v)}\right]=\left\{\frac { 1 } { 2 4 J ^ { 2 } \pi ^ { 2 } N } \left[K\left(3+2 J^{2} \pi^{2}-2 J^{2} \pi^{2} / M\right]\right.\right. \\
+\frac{K}{N} \sum_{\substack{i=1 \\
i \neq j}}^{V}\left(\operatorname{var}\left[I_{X}\left(\Delta f_{i, j} T_{c}\right)\right]+\operatorname{var}\left[I_{Y}\left(\Delta f_{i, j} T_{c}\right)\right]\right) \\
\left.+\frac{N_{0} T_{b}}{4}\right\} \sum_{X} \sum_{q=0}^{M-1}\left[\left(\alpha_{j, q}^{(k, X)}\right)^{2}\right]
\end{array}
$$

\section{BER Performance}

The statistics of the decision metric of a given subcarrier for all users are the same, since each user has the same parameter set. The user superscript is henceforth removed. With BPSK modulation, the bit error probability of subcarrier $j$ is thus

$$
P_{b, j}=Q\left(\sqrt{\frac{\mathrm{E}^{2}\left[Z_{j}\right]}{\operatorname{var}\left[Z_{j}\right]}}\right)=Q\left(\sqrt{2 \gamma_{j}}\right)
$$

where $Q(x)=\int_{x}^{\infty} e^{-t^{2} / 2} d t / \sqrt{2 \pi}$, and $\gamma_{j}$ is the instantaneous signal-to-noise-plus-interference ratio (SNIR) of subcarrier $\mathrm{j}$ which is defined as

$$
\gamma_{j}=\sum_{X} \sum_{q=0}^{M-1} \bar{\gamma}_{j}^{(X)}\left(\alpha_{j, q}^{(X)}\right)^{2}
$$

where $\bar{\gamma}_{j}^{(X)}$ is the average SNIR of the $j$ th subcarrier's LSB or USB RAKE output, given by

$$
\begin{array}{r}
\bar{\gamma}_{j}^{(X)}=\left\{\frac { 1 } { 3 J ^ { 2 } \pi ^ { 2 } N } \left[K\left(3+2 J^{2} \pi^{2}-2 J^{2} \pi^{2} / M\right]\right.\right. \\
\frac{8 K}{N} \sum_{i=1 i \neq j}^{M}\left[V_{X}\left(\Delta f_{i, j} T_{c}\right)+V_{Y}\left(\Delta f_{i, j} T_{c}\right)\right] \\
\left.+\frac{2 N_{0}}{E_{b}}\right\}^{-1}
\end{array}
$$


To obtain the average bit error probability of subcarrier $j$, the instantaneous bit error probability given by (31) should be averaged over the joint pdf of channel tap amplitudes; the joint pdf is the product of the marginal pdfs when the tap amplitudes are independent RVs under the uncorrelated scattering (US) assumption:

$$
\begin{array}{r}
p_{\alpha_{0, L}, \alpha_{1, L}, \cdots \alpha_{M-1, U}}\left(\alpha_{0, L}, \alpha_{1, L}, \cdots \alpha_{M-1, U}\right) \\
=\prod_{X} \prod_{q=0}^{M-1} p_{\alpha_{q, X}}\left(\alpha_{q, X}\right)
\end{array}
$$

The average bit error probability of subcarrier $j$ is thus given by

$$
\begin{array}{r}
\bar{P}_{b, j}=\underbrace{\int_{0}^{\infty} \int_{0}^{\infty} \cdots \int_{0}^{\infty}}_{2 \mathrm{M}-\text { fold }} Q\left(\sqrt{2 \gamma_{j}}\right) \prod_{X} \prod_{q=0}^{M-1} p_{\alpha_{q, X}}\left(\alpha_{q, X}\right) \\
d \alpha_{0, L}, d \alpha_{1, L}, \cdots d \alpha_{M-1, U}
\end{array}
$$

The direct evaluation of (36) requires a $2 M+1$-fold integration, which is difficult to obtain in closed form. To circumvent this difficulty, we use an alternative form of the $Q$ function [20],

$$
Q(x)=\frac{1}{\pi} \int_{0}^{\pi / 2} \exp \left(-\frac{x^{2}}{2 \sin ^{2}(\chi)}\right) d \chi
$$

The alternative $Q$ function form allows us to concisely represent the average $P_{b}$ via the moment generating function (MGF) [20]:

$$
\begin{array}{r}
\bar{P}_{b, j}=\frac{1}{\pi} \underbrace{\int_{0}^{\infty} \ldots \int_{0}^{\infty} \int_{0}^{\pi / 2}}_{2 \mathrm{M}-\text { fold }} \exp \left(-\frac{\sum_{X} \sum_{q=0}^{M-1} \bar{\gamma}_{j}^{(X)}\left(\alpha_{j, q}^{(X)}\right)^{2}}{\sin ^{2} \chi}\right) \\
\times \prod_{X} \prod_{q=0}^{M-1} p_{\alpha_{q, X}}\left(\alpha_{q, X}\right) d \alpha_{0, L}, d \alpha_{1, L}, \cdots d \alpha_{M-1, U} \\
=\frac{1}{\pi} \int_{0}^{\pi / 2} \prod_{X} \prod_{q=0}^{M-1} G\left(\bar{\gamma}_{j, q}^{(X)} \chi\right) d \chi
\end{array}
$$

where $\bar{\gamma}_{j, q}^{X}=\bar{\gamma}_{j}^{X} e^{-\eta q}$ is the average SNIR of the $q$ th RAKE finger output for the LSB or USB RAKE of the $j$ th subcarrier, and $G(x, y)$ is the MGF of the Nakagami- $m$ distribution, [20]

$$
G(\gamma, \chi)=\left(\frac{m \sin ^{2}(\chi)}{m \sin ^{2}(\chi)+\gamma}\right)^{m}
$$

Finally, with the average bit error probability of each subcarrier, the average bit error probability of the proposed system can be obtained via averaging the error probabilities of all subcarriers as $\bar{P}_{b}=\left(\sum_{i=1}^{V} \bar{P}_{b, i}\right) / V$.

\section{NUMERICAL EXAMPLES}

In this section the BER performance of our proposed scheme is compared with that of the MC-DS-CDMA schemes whose average $P_{b}$ is given by equation (47) in [11]. Our comparison here is under the conditions in which all schemes have the same number of subcarriers, the same data rate, the same overall bandwidth, and the same transmission power.

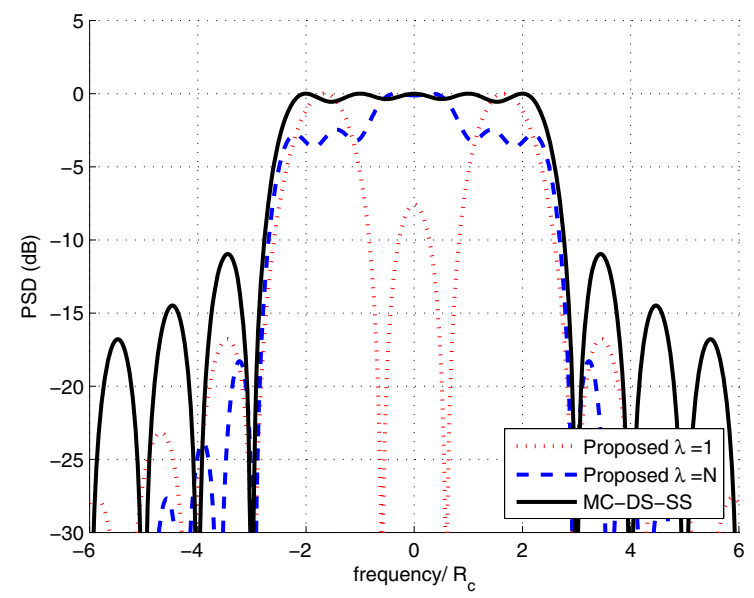

Fig. 4. PSD of proposed scheme and conventional MC-DS-SS with $V=5$, $N_{M C}=64, J=3$, and $N=76$ for $\lambda=1$ and $N=42$ for $\lambda=N$.

Recall that the bandwidth of one sideband signal of our proposed scheme is $2 / T_{c}$, and the frequency separation of the peaks of two sidelobe signals is approximately $J / T_{c}$. Thus, the null-null bandwidth of our proposed scheme is

$$
B W=(J+2) / T_{c}+(V-1) \Delta f
$$

where $\Delta f$ is the frequency separation between the adjacent subcarriers, i.e., $\Delta f=\lambda R_{b}$. The null-null bandwidth of the MC-DS-CDMA with chip duration of $T_{c, M C}$ and frequency separation equal to the chip rate is

$$
B W=2 / T_{c, M C}+(V-1) / T_{c, M C}
$$

Thus under the equal bandwidth and data rate condition, the processing gain relation between the MC-DS-CDMA scheme and our proposed scheme is

$$
N_{M C}=\left\lfloor\frac{(J+2) N+\lambda(V-1)}{V+1}\right\rfloor
$$

The baseband PSD normalized to its maximum value for the proposed scheme with chip waveform parameter $J=3$ and frequency separations of 1 and $\mathrm{N}$ are compared with that of MC-DS-CDMA in Fig. 4. The processing gain for a 5subcarrier MC-DS-CDMA is set to be 64, and the processing gains for our proposed schemes are 76 and 42 respectively for $\lambda=1$ and $\lambda=N$. It can be observed from this figure that $\lambda=1$ and $\lambda=N$ are two extreme examples of spectrum shaping. Specifically, when $\lambda=1$, the intersubcarrier frequency separation is the data rate on each subcarrier, and the PSD of our proposed scheme has spectral nulls around DC; conversely when $\lambda=N$, the PSD of our proposed scheme reaches its maximum value around DC. Thus, the proposed scheme with different frequency separations can be used to adaptively compensate for channel fading when the channel is known at the transmitter. Also worth noting is that the sidelobes of our scheme are more than $17 \mathrm{~dB}$ down, whereas the conventional MC-DS-SS sidelobes are down by approximately $11 \mathrm{~dB}$, hence our scheme suppresses sidelobe levels by approximately $6 \mathrm{~dB}$. Again we note that throughout this paper, the bandwidth to which we refer is the null-to-null bandwidth. This bandwidth measure is used because it is relatively easy to determine. 


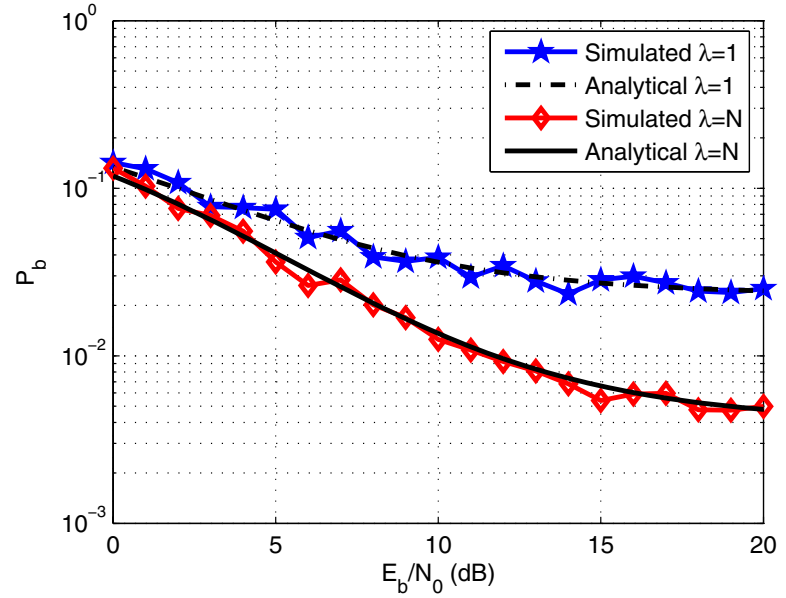

Fig. 5. Analytical and simulated $P_{b}$ vs. $E_{b} / N_{0}$ for $K=10$ users, $V=5$ subcarriers, $N=32$ for proposed scheme with $J=3, \lambda=1, \lambda=N=32$, in Nakagami- $m$ dispersive channel with $m=1, \eta=2 M=5$ channel taps.

In Fig. 5 we show both simulation and analytical results for the systems with $K=10$ users, $V=5$ subcarriers, chip waveform parameter $J=3$, and processing gain of $N=32$ for both $\lambda=1$ and $\lambda=N$. The channel is a Nakagami- $m$ dispersive channel with $m=1$ (Rayleigh fading), PDP decay factor of $\eta=2$, and the number of resolvable paths for each sideband is $M=5$, or a 5-tap channel. From this figure, we can observe that the simulation results match the analytical results, corroborating our standard Gaussian assumption for the interference. The good agreement between the analytical results and simulation results hold for processing gain values greater than 8 . Therefore, in the following examples we use analytical results to compare the BER performance of our proposed scheme with that of MC-DS-CDMA, in which we also consider 10 users, and 5 subcarriers, with processing gain and the number of resolvable channel taps for each subcarrier set to be $N=64$ and $M=5$, respectively. ${ }^{4}$

The average $P_{b}$ of our proposed scheme with $J=3$ and different frequency separations ( $\lambda$ 's), and for MC-DS-CDMA with parameters given previously are shown in Fig. 6 . The processing gains of our proposed schemes are 76, and 42 respectively for $\lambda=1$ and $\lambda=N$. In this example, the channel is a dispersive Nakagami- $m$ channel with $m=1$, $\eta=2$, and the number of channel taps for our proposed scheme with $\lambda=1$ and $\lambda=N$ are respectively 6 , and 4 . From this example we can observe that the $\lambda=N$ intersubcarrier frequency separation case has better performance than that of the $\lambda=1$ case because of the reduced spectral overlapping among subcarriers. In comparison to conventional MC-DS, our proposed scheme with $\lambda=1$ has slightly better performance at SNRs less than $15 \mathrm{~dB}$, and approximately the same performance for SNRs greater than $15 \mathrm{~dB}$.

The effect of the chip waveform parameter $J$ on the BER

\footnotetext{
${ }^{4}$ Note that in Fig. 5, we show that the analytical results match the simulation results for the proposed scheme with two frequency separations without setting the bandwidths of these two configurations to be equal. For the examples in the rest of this section, we compare the BER performance of the proposed scheme with that of the conventional MC-DS-CDMA scheme that has the same number of users, the same number of subcarriers, the same total data rate, the same bandwidth, and the same transmission power.
}

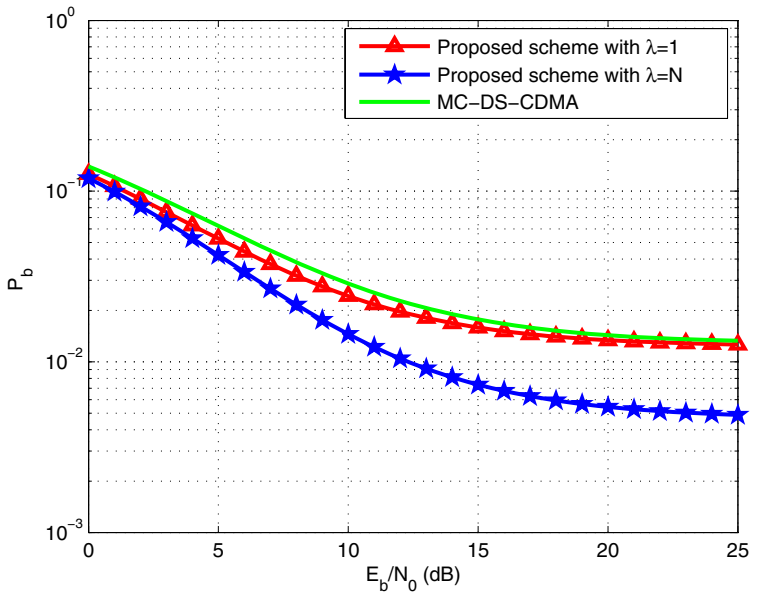

Fig. 6. $\quad P_{b}$ vs. $E_{b} / N_{0}$ for 10 user MC-DS-CDMA system with $V=5$ subcarriers and $N=64$, and proposed scheme with $J=3, \lambda=1, N=76$, and $J=3, \lambda=N=42$, in Nakagami- $m$ dispersive channel with $m=1$, $\eta=2$ and $M=5$ channel taps for MC-DS-CDMA, $M=6$, and 4 for proposed scheme with $\lambda=1$ and $\lambda=N$, respectively.

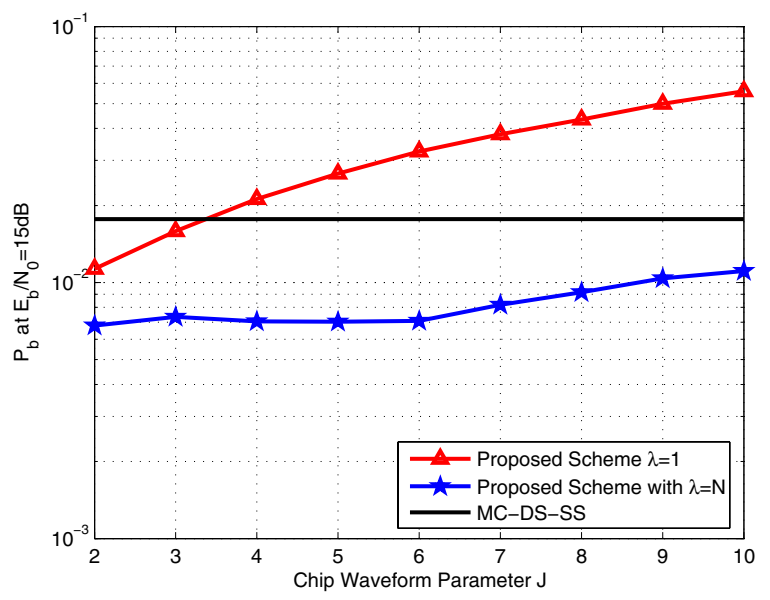

Fig. 7. $\quad P_{b}$ vs. $J$ for 10-user systems over Nakagami- $m$ dispersive channel with $E_{b} / N_{0}=15 \mathrm{~dB}, m=1$, and $\eta=2, V=5$ subcarriers, $N=64$ for MC-DS-CDMA, and $\lambda=1$ and $\lambda=N$ for proposed scheme.

performance of our proposed scheme is shown in Fig. 7. In the figure, the average $P_{b}$ at $E_{b} / N_{0}=15 \mathrm{~dB}$ for our proposed scheme with different values of chip waveform parameter $J$, is compared with that of the conventional MC-DS-CDMA scheme. To ensure the same bandwidth, the processing gain of our proposed scheme with different frequency separations is obtained via equating (40) and (41), and the number of resolvable channel taps is obtained via (9). We can observe in this example that as the value of $\mathbf{J}$ increases, the BER performance of our proposed scheme with $\lambda=1$ degrades whereas the $\lambda=N$ scheme nearly maintains its performance. The reason for the performance degradation is mainly due to the fact that as the value of $J$ increases, the processing gain of our proposed scheme must decrease in order to keep the bandwidth unchanged. For example, the processing gains for the two frequency separations $(\lambda=1$ and $\lambda=N)$ are reduced from 95 and 48, to 31 and 24 , when the value of $J$ increases from 2 to 10 . For the $\lambda=1$ configuration, the spectra of all 


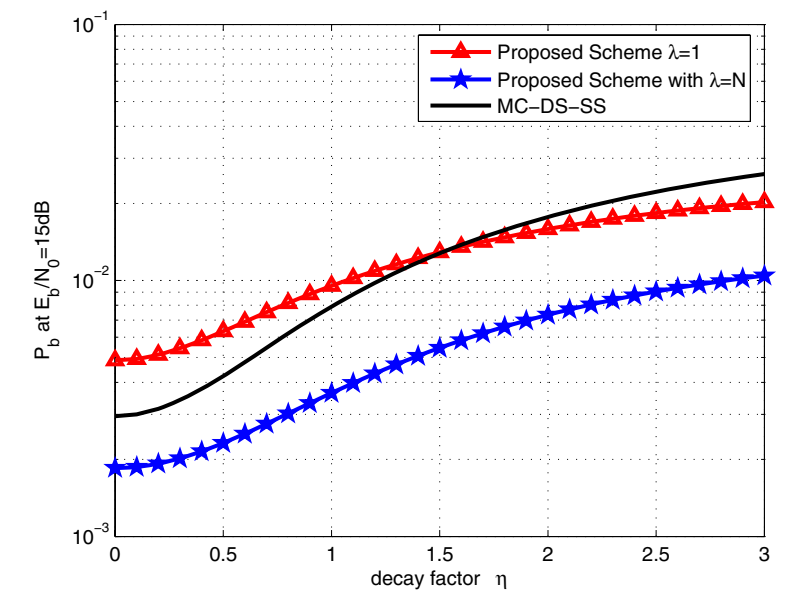

Fig. 8. $P_{b}$ vs. channel power decay factor for 10 -user system with $E_{b} / N_{0}=$ $15 \mathrm{~dB}, N=64, V=5$ subcarriers for MC-DS-CDMA, and for proposed scheme with $J=3, \lambda=1, N=76$, and $J=3, \lambda=N=42$, in Nakagami- $m$ dispersive channel with $m=1$, and $M=5$ for MC-DSCDMA, $M=6$, and 4 for proposed scheme with $\lambda=1$ and $\lambda=N$, respectively.

subcarriers overlap significantly, thus the loss in processing gain reduces its capability to suppress interference from other subcarriers. On the other hand, only the spectra of adjacent subcarriers overlap for the $\lambda=N$ configuration, yielding less intersubcarrier interference. As a result, the reduction of processing gain for the $\lambda=N$ scheme does not as strongly affect the average $P_{b}$.

We next show the BER performance of our proposed scheme in different channel conditions. The average $P_{b}$ at $E_{b} / N_{0}=15 \mathrm{~dB}$ with $K=10$ users for our proposed scheme with $J=3$ and for MC-DS-CDMA, with different decay factors, is shown in Fig. 8. The channel is a Nakagami- $m$ fading channel with $m=1$, and the decay factor $\eta$ varies from 0 to 3 . We assume the receiver is capable of capturing all the channel taps, i.e., we employ a 5-finger RAKE for one subcarrier of MC-DS-CDMA, and 4-finger and 6-finger RAKEs for each sideband of our proposed schemes with $\lambda=1$ and $\lambda=N$ respectively. The results shown in Fig. 8 indicate that as the decay factor increases, the average $P_{b}$ of all three system increases. This agrees with the fact that RAKE has the best performance for a uniform PDP. The proposed scheme with $\lambda=N$ outperforms the reference system at any value of $\eta$ and when $\eta>1.8$, the proposed scheme with $\lambda=1$ has a better performance than that of MC-DS-CDMA. As the decay factor increases, the majority of the energy is contained within the first few (lower delay) channel taps. Thus, when these lower-delay taps incur deep fading, the SNIR output of the MRC is low even if the low-energy, higher-delay channel taps are not faded. For our proposed scheme, however, the channels for LSB and USB are independent identical channels. Therefore, the probability of both sideband signals undergoing a deep fade is greatly reduced.

In Fig. 9 the average $P_{b}$ as a function of the Nakagami$m$ factor is shown for the three schemes. The parameters of conventional MC-DS-CDMA and for our proposed schemes with $\lambda=1$ and $\lambda=N$ are the same as in the previous

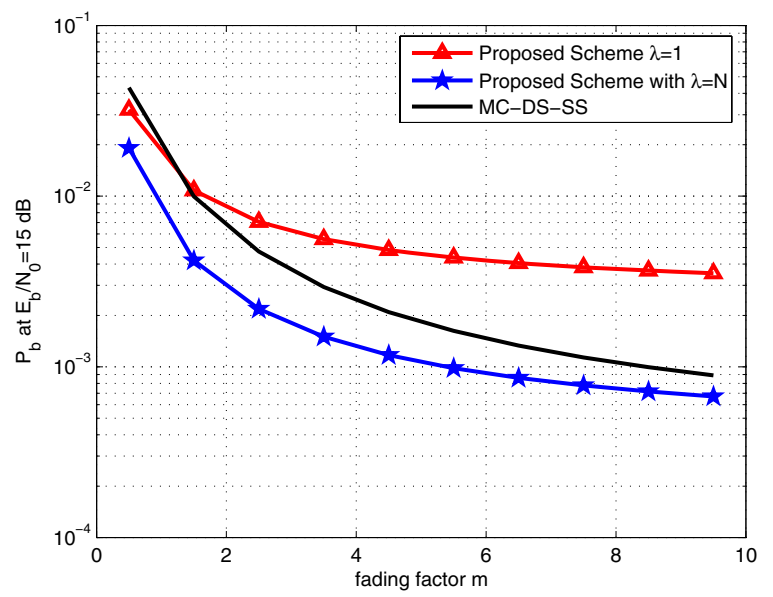

Fig. 9. $P_{b}$ vs. channel $m$ for 10 -user system with $E_{b} / N_{0}=15 \mathrm{~dB}$, $N=64, V=5$ subcarriers for MC-DS-CDMA, and for proposed scheme with $J=3, \lambda=1, N=76$, and $J=3, \lambda=N=42$, in Nakagami- $m$ dispersive channel with $\eta=2$, and $M=5$ for MC-DS-CDMA, $M=6$, and 4 for proposed scheme with $\lambda=1$ and $\lambda=N$, respectively.

example, and the channel is similar to that of the previous example except that instead of varying the decay factor $\eta$, here we fix the decay factor to be $\eta=2$ and allow the fading parameter $m$ to vary from $1 / 2$ to 10 . As we know, $m=1 / 2$ is the so called one-sided Gaussian distribution-a severe case of fading. As $m$ increases, the channel is less severely faded, and this example shows the effect of the diversity via LSB and USB combining in our proposed scheme.

As can be observed from this figure, the average bit error probabilities of MC-DS-CDMA and those of our proposed schemes decrease as the value of $m$ increases, as expected. Both our proposed schemes with $\lambda=1$ and $\lambda=N$ outperform MC-DS-CDMA at $m=1 / 2$. As the value of $m$ increases, the performance of the $\lambda=1$ scheme worsens relative to that of conventional MC-DS-CDMA, whereas the $\lambda=N$ scheme still has better performance than that of the MC-DS-CDMA scheme. The reason is that when the channel fading is milder ( $m$ increases), the dominant effect on performance becomes the MAI; in this case, since diversity is used to combat fading instead of suppressing MAI, the diversity introduced by combining LSB and USB signals in our scheme is less effective. As a result, the performance of the $\lambda=N$ scheme approaches that of MC-DS-CDMA. This example and the previous one show that the proposed scheme can be used in environments where the wireless channel is severely faded, i.e., a large PDP decay factor $\eta$ and a small $m$ value.

The average $P_{b}$ of our proposed scheme at $E_{b} / N_{0}=15$ $\mathrm{dB}$ with $\lambda=1$ and $\lambda=N$ and that of conventional MCDS-CDMA are shown in Fig. 10. The parameters of all three schemes are the same as in the previous examples, except that we vary the number of subcarriers from 3 to 20 , and adjust each subcarrier's processing gain of our proposed scheme to make sure that our proposed schemes and the conventional MC-DS-CDMA with a processing gain of $N=64$ have the same bandwidth. The channel is a Nakagami- $m$ fading channel with $m=1, \eta=2$ and the number of resolvable channel taps for conventional MC-DS-CDMA is $M=5$, 


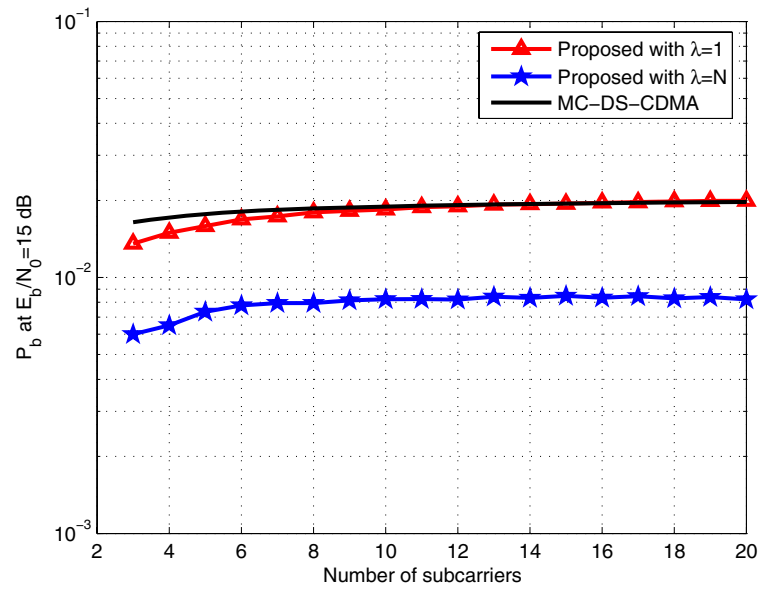

Fig. 10. $P_{b}$ vs. number of subcarriers for 10-user system with $E_{b} / N_{0}=15$ $\mathrm{dB}, N=64$ for MC-DS-CDMA, and proposed scheme with $J=3, \lambda=$ 1 , and $J=3, \lambda=N=42$, in Nakagami- $m$ dispersive channel with $m=1$ $\eta=2$, and $M=5$ for MC-DS-CDMA.

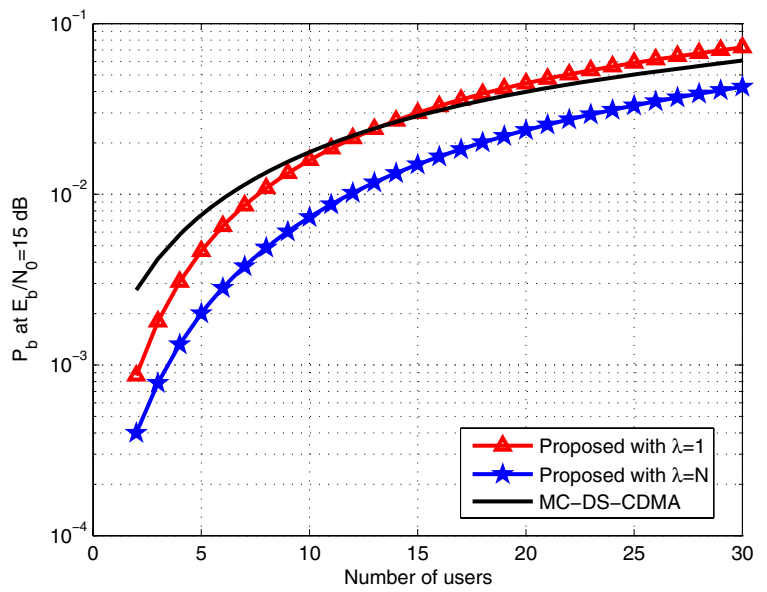

Fig. 11. $P_{b}$ vs. number of users with $E_{b} / N_{0}=15 \mathrm{~dB}, N=64, V=5$ subcarriers for MC-DS-CDMA and proposed scheme with $J=3, \lambda=1$, $N=76$, and $J=3, \lambda=N=42$, in Nakagami- $m$ dispersive channel with $m=1 \eta=2$, and $M=5$ for MC-DS-CDMA, $M=6$, and 4 for proposed schemes with $\lambda=1$ and $\lambda=N$, respectively.

and the number of resolvable channel taps for our proposed schemes are given by (9). As demonstrated in this figure, the error performance of all three schemes is insensitive to the number of subcarriers, $V$, when $V$ is greater than some value. Specifically for the parameters in the figure, when $V$ is greater than 6 , the curves are flat as the number of subcarriers increases. In addition, the error performance of our proposed scheme with $\lambda=1$ is similar to that of conventional MCDS-CDMA when the number of subcarriers is large because of the increased interference caused by strongly overlapped subcarriers; conversely, since the adjacent subcarriers overlap at their spectral nulls in our proposed scheme with $\lambda=N$, this scheme has lower bit error probabilities despite the increase in the number of subcarriers.

The influence of different system load (number of users) on the average $P_{b}$ is shown in Fig. 11. The parameters of conventional MC-DS-CDMA and for our proposed schemes

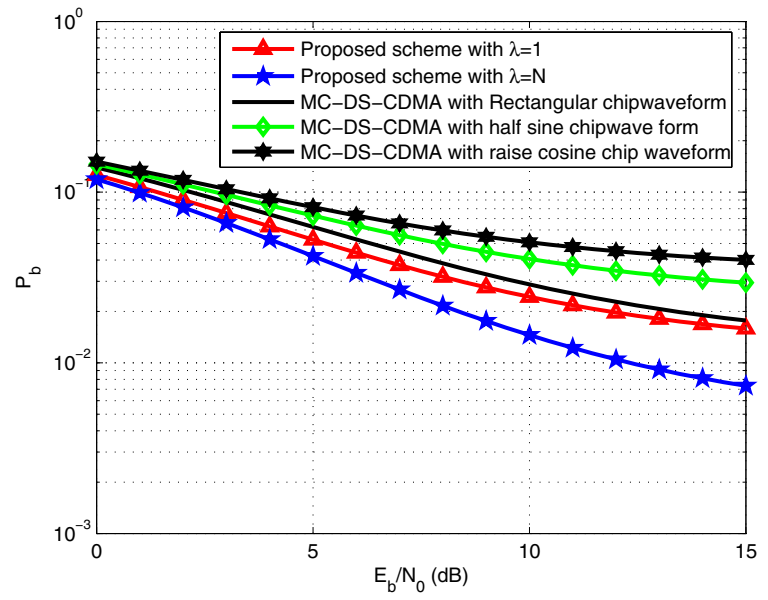

Fig. 12. $\quad P_{b}$ vs. $E_{b} / N_{0}$ for 10 user MC-DS-CDMA system with $V=5$ subcarriers $N=64,43$ and 32 respectively for rectangular, half-sine and raised-cosine chip waveforms, proposed scheme with $J=3, \lambda=1, \mathrm{~N}=76$, and $J=3, \lambda=N=42$, in Nakagami- $m$ dispersive channel with $m=$ $1, \eta=2$ and $M=5$ channel taps for MC-DS-CDMA, $M=6$, and 4 for proposed scheme with $\lambda=1$ and $\lambda=N$, respectively.

with $\lambda=1$ and $\lambda=N$ and the channel are the same as in the example shown in Fig. 6. We show the average $P_{b}$ of three systems with different numbers of active users. As expected, with more users added to the system, the error performance of all three schemes degrades, and the performance of our proposed schemes with $\lambda=1$ and $\lambda=N$ approaches that of conventional MC-DS-CDMA as the number of users increases. This is due to reasons similar to those of the example in Fig. 10. Specifically, as the number of user increases, MAI becomes the dominant factor in performance loss, and the diversity introduced by combining the LSB and USB signals in our scheme is less effective.

We also compare the error performance of our scheme with the conventional MC-DS-CDMA with the half sine and raised cosine chip waveforms of [17] in Fig. 12. The parameters of conventional MC-DS-CDMA with the rectangular chip waveform, our proposed schemes, and the channel are the same in previous example. The processing gains of MCDS-CDMA with chip waveforms other than rectangular are adjusted to ensure the same bandwidth for all schemes. Specifically, with the spectral expansion of the half sine and raised cosine chip waveforms [17] considered, the processing gains of MC-DS-CDMA with these two chip waveforms are respectively 43 and 32 . The performance ranking shown in this figure-our proposed scheme with $\lambda=N$ followed by the proposed scheme with $\lambda=1$, conventional MC-DS-CDMA with rectangular chip waveform, MC-DS-CDMA with the half sine chip waveform, and MC-DS-CDMA with the raised cosine chip waveform ${ }^{5}$ - suggests that our proposed scheme with $\lambda=N$ yields the maximum diversity gain.

\footnotetext{
${ }^{5}$ The authors of [17] reached the conclusion that the MC-DS-CDMA with rectangular, half sine, and raised cosine chip waveform have similar error performances without equating the bandwidth of MC-DS-CDMA with these chip waveforms.
} 


\section{CONCLUSION}

A new modulation scheme that shapes the spectrum of an MC-DS-SS signal via sinusoidal chip waveforms was proposed. This sinusoidal chip shaping enables generation of distinct upper and lower sideband component signals for each subcarrier, which can be exploited for frequency diversity. The BER of this spectrally shaped MC-DS-CDMA with dual sideband combining was considered over Nakagami-m dispersive fading channels. We investigated the effects of chip waveform index, PDP shape, and the channel fading conditions on the performance of the proposed scheme. With properly selected parameters, the BER performance of the proposed scheme is better than that of an equivalent MC-DS-SS signal with rectangular, half sine, and raised cosine chip waveforms. Future work includes investigating the performance of our proposed scheme when the two sidebands incur correlated fading, and the effects of Doppler.

\section{APPENDIX I}

By substituting the chip waveform and discarding the double frequency term, 4) can be written as

$$
\begin{aligned}
& \int_{0}^{T_{b}} \sum_{n=0}^{N-1} p^{2}\left(t-n T_{c}\right) \cos \left(2 \pi f_{i} t\right) \cos \left(2 \pi f_{j} t\right) d t \\
& =\sum_{n=0}^{N-1} \int_{n T_{c}}^{(n+1) T_{c}} \sin ^{2}\left(\frac{J \pi}{T_{c}} t\right) \cos \left(2 \pi \Delta f_{i, j} t\right) d t
\end{aligned}
$$

where $\Delta f_{i, j}=\left|f_{i}-f_{j}\right|$. With some straight forward algebra, it can be shown that when $\Delta f_{i, j} \neq J$ (43) can be expressed as

$$
\frac{J^{2} \sin \left(\pi \Delta f_{i, j} T_{c}\right)}{2 \pi \Delta f_{i, j} T\left[J^{2}-\left(\Delta f_{i, j} T\right)^{2}\right]} \sum_{n=0}^{N-1} \cos \left[\pi \Delta f_{i, j} T_{c}(2 n+1)\right]
$$

Following the identity [21]

$$
\sum_{m=0}^{N-1} \cos (m y+x)=\frac{\cos \{x+[(N-1) / 2] y\} \sin (N y / 2)}{\sin (y / 2)}
$$

(44) reduces to

$$
\frac{J^{2} \cos \left(\pi N \Delta f_{i, j} T_{c}\right) \sin \left(\pi N \Delta f_{i, j} T_{c}\right)}{2 \pi \Delta f_{i, j} T\left[J^{2}-\left(\Delta f_{i, j} T\right)^{2}\right]}
$$

which equals zero when $\Delta f_{i, j}=\lambda /\left(N T_{c}\right)=\lambda R_{b}$. Following the same method, it can be shown that when $\Delta f_{i, j}=J$, (43) reduces to $T_{b} / 4$, not zero. Thus, in order to make sure that subcarrier $i$ and subcarrier $j$ are orthogonal, we require $\Delta f_{i, j}=\left|f_{i}-f_{j}\right|=\lambda / T_{b}$ and $\Delta f_{i, j} \neq J$.

\section{APPENDIX II}

We derive the variance of the integrals in (25) and (26). Denote the integral in (25) as $I_{X}\left(\Delta f_{i, j} T_{c}\right)$.

$$
I_{X}\left(\Delta f_{i, j} T_{c}\right)=\int_{0}^{\varepsilon} E_{i, X}(t-\varepsilon, 0) E_{j, X}(t, 0) d t
$$

With the definition of $E_{i, X}$, it is easy to show that (47) can be expressed as

$$
I_{X}\left(\Delta f_{i, j} T_{c}\right)=\frac{1}{2} \int_{0}^{\varepsilon} \cos \left(2 \pi \Delta f_{i, j} t+\frac{J \pi}{T_{c}} \varepsilon+\theta\right) d t
$$

where $\theta=\phi+2 \pi f_{i} \varepsilon-J \pi \varepsilon / T_{c}$ is the aggregate phase due to the delay and the channel, uniformly distributed in $[0,2 \pi)$. Note that in (48) we have discarded the term containing the high frequency component at frequency $f_{i}+f_{j}$. Upon averaging the variable given by (48) over the aggregate phase $\theta$ and the fractional chip part of delay, $\epsilon$ the variance of $I_{x}$ is

$$
\operatorname{var}\left[I_{X}\left(\Delta f_{i, j} T_{c}\right)\right]=\frac{T_{c}^{2}}{32} \frac{2 \pi \Delta f_{i, j} T_{c}-\sin \left(2 \pi \Delta f_{i, j} T_{c}\right)}{\pi \Delta f_{i, j} T_{c}}
$$

When $\Delta f_{i, j}=0$, representing the same-subcarrier samesideband interference, the variance of $I_{X}\left(\Delta f_{i, j} T_{c}\right)$ is

$$
\operatorname{var}\left[I_{X}\left(\Delta f_{i, j} T_{c}\right)\right]=\frac{T_{c}^{2}}{24}
$$

Following the same method, the integral in the cross correlation function between different sideband signals in (19) is denoted by $I_{Y}\left(\Delta f_{i, j} T_{c}\right)$, and when $\Delta f_{i, j} T_{c} \neq J$ its variance is

$$
\operatorname{var}\left[I_{Y}\left(\Delta f_{i, j} T_{c}\right)\right]=\frac{T_{c}^{2}}{16} \frac{2 X-\sin (2 X)}{X^{3}}
$$

where $X=\pi\left(\Delta f_{i, j} T_{c}-J\right)$. When $\Delta f_{i, j} T_{c}=J$, its variance is

$$
\operatorname{var}\left[I_{Y}\left(\Delta f_{i, j} T_{c}\right]=\frac{T_{c}^{2}}{24}\right.
$$

\section{REFERENCES}

[1] R. L. Pickholtz, D. L. Schilling, and L. B. Milstein, "Theory of spread-spectrum communications-a tutorial," IEEE Trans. Commun. vol. COM-30, no. 5, pp. 855-884, May 1982.

[2] S. Hara and R. Prasad, "Overview of multicarrier CDMA," IEEE Commun. Mag., vol. 35, no. 12, pp. 126-133, Dec. 1997.

[3] — , "Design and performance of multicarrier CDMA system in frequency-selective rayleigh fading channels," IEEE Trans. Veh. Technol., vol. 48, no. 5, pp. 1584-1595, Sept. 1999.

[4] X. Gui and T. Ng, "Performance of asynchronous orthogonal multicarrier CDMA systems in frequency selective fading channel," IEEE Trans. Commun., vol. 47, no. 7, pp. 1084-1091, July 1999.

[5] E. A. Sourour and M. Nakagawa, "Performance of orthogonal multicarrier CDMA in a multipath fading channel," IEEE Trans. Commun. vol. 44, pp. 356-366, Mar. 1996.

[6] S. Kondo and L. B. Milstein, "Performance of multicarrier DS-CDMA system," IEEE Trans. Commun., vol. 44, no. 2, pp. 238-246, Feb. 1996.

[7] L. Vandendorpe, "Multitone spread spectrum multiple access communications system in a multipath rician fading channel," IEEE Trans. Veh. Technol., vol. 44, no. 2, pp. 327-337, May 1995.

[8] K. Yip, X. Zhang, T. S. Ng, and J.Wang, "On the multiple-access capacity of Multitone-CDMA communications," IEEE Commun. Lett., vol. 4, no. 2, pp. 40-42, Feb. 2000.

[9] I. Sen and D. W. Matolak, "Reduced-complexity bandwidth efficient multitone direct sequence spread spectrum," in Proc.2004 IEEE Sarnoff Symp. on Advances in Wired and Wireless Comm., Princeton, NJ, Apr. 2004, pp. 26-27.

[10] H. Li and D. W. Matolak, "Phase noise and fading effects on system performance in MT-DS-SS," IEEE Trans. Veh. Technol., vol. 54, no. 2 , pp. 1759-1767, 2006.

[11] L.-L. Yang and L. Hanzo, "Performance of generalized multicarrier DS-CDMA over Nakagami-m fading channels," IEEE Trans. Commun., vol. 50, no. 6, pp. 956-966, June 2002.

[12] C. W. You and H. D.S., "Multicarrier CDMA systems using time-domain and frequency-domain spreading codes," IEEE Trans. Commun., vol. 51, no. 1, pp. 17-21, Jan. 2003.

[13] D. W. Matolak and W. Xiong, "Spectrally shaped generalized multitone direct sequence spread spectrum," IEEE Trans. Veh. Technol., vol. 55, no. 4, pp. 1224-1238, 2006.

[14] J. S. Lehnert, "Chip waveform selection in offset-quaternary directsequence spread-spectrum multiple-access communications," master's thesis, Univ. of Illinois, Urbana, IL, 1981.

[15] M. A. Landolsi and W. E. Stark, "DS-CDMA chip waveform design for minimal interference under bandwidth, phase, and envelope constraints," IEEE Trans. Commun., vol. 47, no. 11, pp. 1737-1749, Nov. 1999. 
[16] H. H. Nguyen, "An improved design of chip waveforms for band-limited DS-CDMA systems," IEEE Trans. Veh. Technol., vol. 53, no. 5, pp. 1379-1386, 2004.

[17] L. Yang and L. Hanzo, "An improved design of chip waveforms for band-limited DS-CDMA systems," IEEE Trans. Veh. Technol., vol. 51, no. 5, pp. 748-752, May 2003.

[18] H. H. Nguyen, "Effect of chip waveform shaping on the performance of multicarrier CDMA systems," IEEE Trans. Veh. Technol., vol. 54, no. 3, pp. 1022-1029, May 2005.

[19] J. G. Proakis, Digital Communications, 4th ed. New York: McGrawHill, 2000.

[20] M.-S. Alouini and A. J. Goldsmith, "A unified approach for calculating error rates of linearly modulated signals over generalized fading channels," IEEE Trans. Commun., vol. 47, pp. 1324-1334, 1999.

[21] R. L. Peterson, R. E. Ziemer, and D. E. Borth, Introduction to Spread Spectrum Communications. Upper Saddle River, NJ: Prentice-Hall, 1995.

Wenhui Xiong (SM'03) was born in Chengdu, China, and received his B.S. and M.S. degrees from the University of Electronic Science and Technology of China, Chengdu, China, in 1999 and 2002, and the Ph.D Degree from Ohio University, Athens, $\mathrm{OH}$, in 2007 all in electrical engineering. He is currently working as a senior engineer at corporate R\&D group, Qualcomm inc., where he is working on mobile positioning for CDMA network.

David W. Matolak (M'83-SM'00) was born in Johnstown, PA, and received the B.S. degree from The Pennsylvania State University, University Park, PA, in 1983, the M.S. degree from The University of Massachusetts, Amherst, MA, in 1987, and the Ph.D. degree from The University of Virginia, Charlottesville, VA, in 1995, all in electrical engineering.
He was with the Rural Electrification Administration, Washington, D.C., from 1983 to 1985 , and worked on upgrading specialized rural telecommunication systems. From 1985 to 1986 , he was with the UMass LAMMDA Laboratory, where he worked on the full-wave analysis, design, fabrication, and testing of planar microwave transmission lines and antennas. From 1986 to 1989 he was with AT\&T Bell Laboratories, in the Microwave Radio Systems Development Department, where he worked on analytical and empirical characterization of nonlinearities and their effect on QAM transmission. In 1990, he joined the University of Virginia's Communication Systems Laboratory, where he researched trellis coding and equalization for TDMA mobile radio systems. From 1994 to 1996, he was with Lockheed Martin Tactical Communication Systems, where he was Lead System Engineer on the development of a wireless local loop synchronous CDMA communication system. From 1996 to 1998, he was with the MITRE Corporation, where he worked on the analysis and modeling of various digital radio communication systems. He was with Lockheed Martin Global Telecommunications from 1998 to 1999, and worked on mobile satellite communication system analysis and design. In September 1999, he joined the School of Electrical Engineering and Computer Science at Ohio University, Athens, Ohio. His research interests are communication over fading channels, radio channel modeling, multicarrier transmission, and CDMA.

Prof. Matolak is a member of Eta Kappa Nu, and Sigma Xi. He has served on served on multiple IEEE conference technical program committees, and was also chair of the Geo Mobile Radio Standards group in the Telecommunications Industries Association's (TIA's) Satellite Communications Division. 